\title{
25 Research Square \\ Testicular Hemangioma in a Child: a Rare Case Report and Review of Literature
}

\section{Kaiping Zhang}

Anhui provincal children hospital

Ye Zhang

Anhui Provincial Children's Hospital

Yin Zhang

Anhui Provincial Children's Hospital

Min Chao ( $\nabla$ cm0654@sina.com )

Anhui Provincial Children's Hospital https://orcid.org/0000-0002-1995-0431

Case report

Keywords: Testicular hemangioma, Child, Case report

Posted Date: June 23rd, 2021

DOl: https://doi.org/10.21203/rs.3.rs-635501/v1

License: (c) (1) This work is licensed under a Creative Commons Attribution 4.0 International License. Read Full License 


\section{Abstract}

Background: Testicular hemangioma is a extremely rare and typically occurs in childhood or adolescence. It is a benign tumor that appears as a slowly growing painless mass.

Case presentation: We herein present the case of a 3 year-old male patient with testicular hemangioma accompanying hydrocele, who underwent surgical resection.

Conclusions: This article discusses the diagnosis and currently available treatment options of testicular hemangioma. It also can be provide clinical experiences for urologist in face of similar situation.

\section{Introduction}

Testicular hemangioma are rare benign vascular tumors that arise from the inner layer of the tunica albuginea [1]. It was commonly seen in infants and children without any discomfort. Some cases are poorly defined and indistinguishable from malignant tumours. To date, only a few studies were reported on testicular hemangioma in the literature. We herein present the case of a 3 year-old male patient with hydrocele accompanying right testicular hemangioma. Then, testicular-sparing surgery can be performed for definitive treatment in patient. He is recovered well after 3 months of follow-up. This article discusses the diagnosis and currently available treatment options of testicular hemangioma.

\section{Case Presentation}

We reported the case of a 3 year-old male patient with hydrocele accompanying right testicular hemangioma. Physical examination revealed a painless scrotal mass with a positive transillumination test. Initial laboratory studies including urinalysis, urine culture, complete blood count, and so on were negative. The levels of preoperative serum tumor markers are normal. A ultrasound scan of the scrotum revealed a solid mass located in the upper pole of the right testis, $19 \mathrm{~mm}$ in diameter with slightly decreased echogenicity and regular contours (Fig. 1A). Doppler scans demonstrated a dense network of blood vessels and increased blood flow in the lesion described (Fig. 1B). The patient underwent laparscopic exploration, and then open surgery was performed through an inguinal approach. Taking the suspected malignancy into account, a decision was made to remove the solid mass using the inguinal approach. The specimen was sent to do a high-speed frozen section histodiagnosis and found it was hemangioma (Fig. 2). In order to preservation of a healthy tissue margin, resection of the solid mass was performed in patient. Then, the wound was stitched up. histopathological examination of the whole specimen revealed a multifocal hemangioma of the testis accompanying proliferation.

Immunohistochemistry: vimentin (+), CD31 (+), CD34 (+), Ki-67(+8\%), CK(-), calretinin (-). The patient was discharged on day 4 after surgery, and recovered well after postoperative 3 months. Bilateral scrotal hydrocele was found at the age of 2 in the patient's medical history, and the ligation of peritoneo-vaginal duct was performed by laparoscopic technique. 


\section{Discussion}

Testicular tumors are predominantly malignant in adults [2]. In some cases, it is difficult to discriminate benign tumor from malignancy in terms of the diagnosis and treatment. Generally, radical orchidectomy remains the best choice for patients with malignancy, whereas treatment option of benign testicular tumors is testis-sparing approach. The preoperative differentiation is important for clinicians who encounter a testicular mass. Hemangioma is a rare benign tumor of the testicle that arise from the inner layer of the tunica albuginea, which often be confronted with a significant diagnostic and therapeutic dilemma [3]. This may be in part because testicular hemangioma have not been extensively reported in the literature. In addition, pediatric testicular tumors are distinct from adult testicular tumors. Whereas only a small number of adult testicular tumors are benign, approximately one-third of pediatric testicular tumors are benign [4]. That suggests that prepubertal tumors should be managed in a manner different from adult. We present this rare case in a child, and discuss the diagnosis and currently treatment options of testicular hemangioma.

Most testicular hemangiomas present as painless but palpable lesions similar to malignant tumors on clinical presentation [5]. Appropriate pre-operative and intra-operative recognition of tumors is vital for treatment options. Ultrasonography is a commonly useful tool to diagnose hemangioma in clinic. Testicular hemangioma often presents as hypo-echogenic central part with hyperechogenic findings [6]. Although ultrasonography has been applied successively to the diagnose of testicular hemangioma, surgical exploration and pathology are still required for definitive diagnosis [7]. Occasionally magnetic resonance imaging (MRI) could distinguish benign tumors from malignant testicular tumors. However, reports on the use of MRI in the diagnosis of testicular hemangiomas are rare. T1- and T2-weighted images did not allow to determine whether the tumor was benign or malignant [8]. Clinical examination and imaging studies do not often suffice to get a correct diagnosis. Histological examination with immunohistochemical staining contributes to making a correct and ultimate diagnosis. Immunohistochemically, the vascular origin of the tumor often shows positive expressions of vimentin, CD31, CD34, and Vimentin, and negative expressions of CK, calretinin, which is helpful for differential diagnosis. Serum tumour markers are normal.

As for testicular malignancy, the standard course of surgery mainly includes, via the inguinal approach, cutting off the spermatic cord at the level of the internal inguinal ring. If you make doubt for malignancy of the testis, intraoperative frozen sections may be recommended from the lesion, or the whole lesion together with a margin of healthy testis [9]. If the diagnosis is not definitive during operation, orchidectomy must be performed. If a benign lesion is confirmed intraoperatively, testicular-sparing surgery can be performed for definitive treatment instead of a radical orchiectomy [10].

Current therapeutic modalities of testicular hemangioma include surgical excision, laser fulguration, intralesional sclerotherapy and cryotherapy. Complete local excision and follow up are widely used for testicular hemangioma. Because intraoperative frozen section examination could be performed, testicular sparing surgery is possible, especially for patients with negative tumor marker findings. Meanwhile, in 
order to protect fertility, we also should take testicular sparing surgery into consideration first [11]. Testis sparing surgery is commonly done in pre-pubescent tumors as the proportion of benign tumors is relatively higher than in adults [12]. Local recurrence occurs in some patients but there are no reports of distant metastases. To date, testicular hemangiomas, managed by either radical or partial orchiectomy, have not yet shown any malignant potential. Sometimes the rapid growth of the testicular hemangioma has been previously reported and might be explained by the development of wide, intra-tumoural haemorrhage [12].

Testicular hemangiomas are rare benign vascular tumors. We performed testicular-sparing surgery in our case. In this way, we aimed to protect the baby from hypogonadism, infertility, and poor cosmesis. However, long-term follow-up is needed.

\section{Abbreviations}

MRI: Magnetic Resonance Imaging.

\section{Declarations}

\section{Acknowledgement}

Not applicable.

\section{Authors' contributions}

$\mathrm{MC}$ and $\mathrm{YZ}$ conceived of this letter and led the development of the letter to the editor. $\mathrm{KZ}$ wrote the first draft of the letter, and coordinated and integrated comments. $K Z$ and $Y Z$ critically revised and edited successive drafts of the manuscript. All authors read and approved the final manuscript.

\section{Funding}

Not applicable.

\section{Availability of data and materials}

Not applicable.

\section{Ethics approval and consent to participate}

Written informed consent was obtained from the patient' parent for publication of this case report and accompanying images.

\section{Consent for publication}

Written informed consent for publication was obtained from all participants. 


\section{Competing interests}

The authors declare that they have no competing interests.

\section{References}

1. Ghoreifi A, Djaladat H. Management of Primary Testicular Tumor. Urol Clin North Am. 2019;46:333-9.

2. Gilligan T, Lin DW, Aggarwal R, et al. Testicular Cancer, Version 2.2020, NCCN Clinical Practice Guidelines in Oncology. J Natl Compr Canc Netw. 2019;17:1529-54.

3. Liu B, Chen J, Luo J, Zhou F, Wang C, Xie L. Cavernous hemangioma of the testis mimicking a testicular teratoma. Exp Ther Med. 2013;6:91-2.

4. Lee SD. Korean Society of Pediatric Urology. Epidemiological and clinical behavior of prepubertal testicular tumors in Korea. J Urol. 2004;172:674-8.

5. Kryvenko ON, Epstein JI. Testicular hemangioma: a series of 8 cases. Am J Surg Pathol. 2013;37:860e6.

6. Sheu GL, Hammer Y, Kirsch AJ. Testicular capillary hemangioma presenting as an incidental contralateral lesion in a child with cryptorchidism. Urology. 2012;80:1135-7.

7. Spiesecke P, Fischer T, Stephan C, Maxeiner A, Hamm B, Lerchbaumer M. Multiparametric Ultrasound (mpUS) of a Rare Testicular Capillary Hemangioma. Case Rep Radiol. 2019;2019:7568098.

8. Zaidi SN, Fathaddin AA. Testicular capillary hemangioma-a case report of a rare tumor. Indian J Pathol Microbiol. 2012;55:557-9.

9. Bujons A, Caffaratti J, Pascual M, Angerri O, Garat JM, Villavicencio H. Testicular tumours in infancy and children. Actas Urol Esp. 2011;35:93-8.

10. Passman C, Urban D, Klemm K, Lockhart M, Kenney P, Kolettis P. Testicular lesions other than germ cell tumours: feasibility of testis-sparing surgery. BJU Int. 2009;103:488e91.

11. Rolle L, Tamagnone A, Destefanis $P$, et al. Microsurgical "testis-sparing" surgery for nonpalpable hypoechoic testicular lesions. Urology. 2006;68:381-5.

12. Bonetti LR, Schirosi L, Sartori G, et al. Rapidly-growing hemangioma of the testicle clinically simulating an aggressive neoplasm. A case report, Pathologica. 2009;101:123-5

\section{Figures}




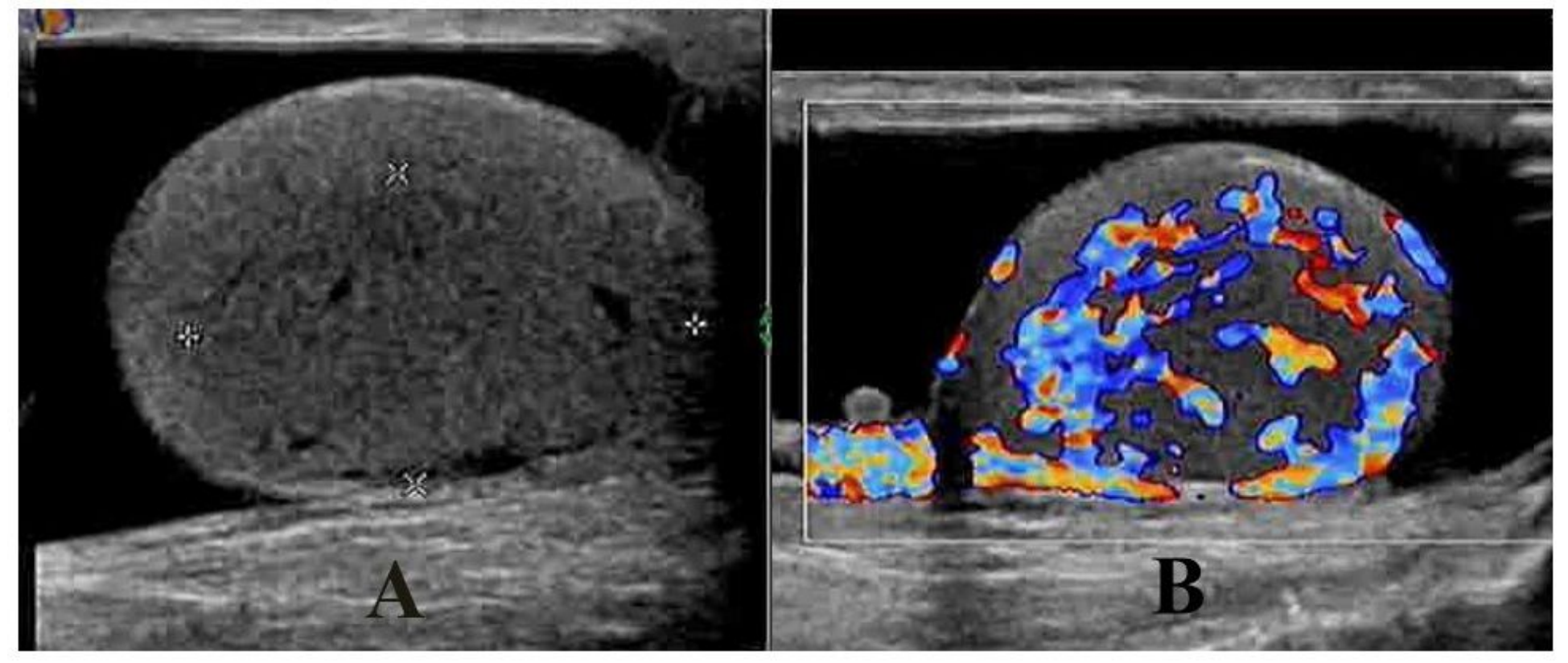

\section{Figure 1}

Testicular hemangioma in a 3-year-old male child. (A) Ultrasound images of the right testicle show a hypoechoic intratesticular mass, measuring $1.9 * 1.4 * 1.2 \mathrm{~cm}$. (B) Color Doppler ultrasound image of the right testicle demonstrates blood flow within the intratesticular mass. 


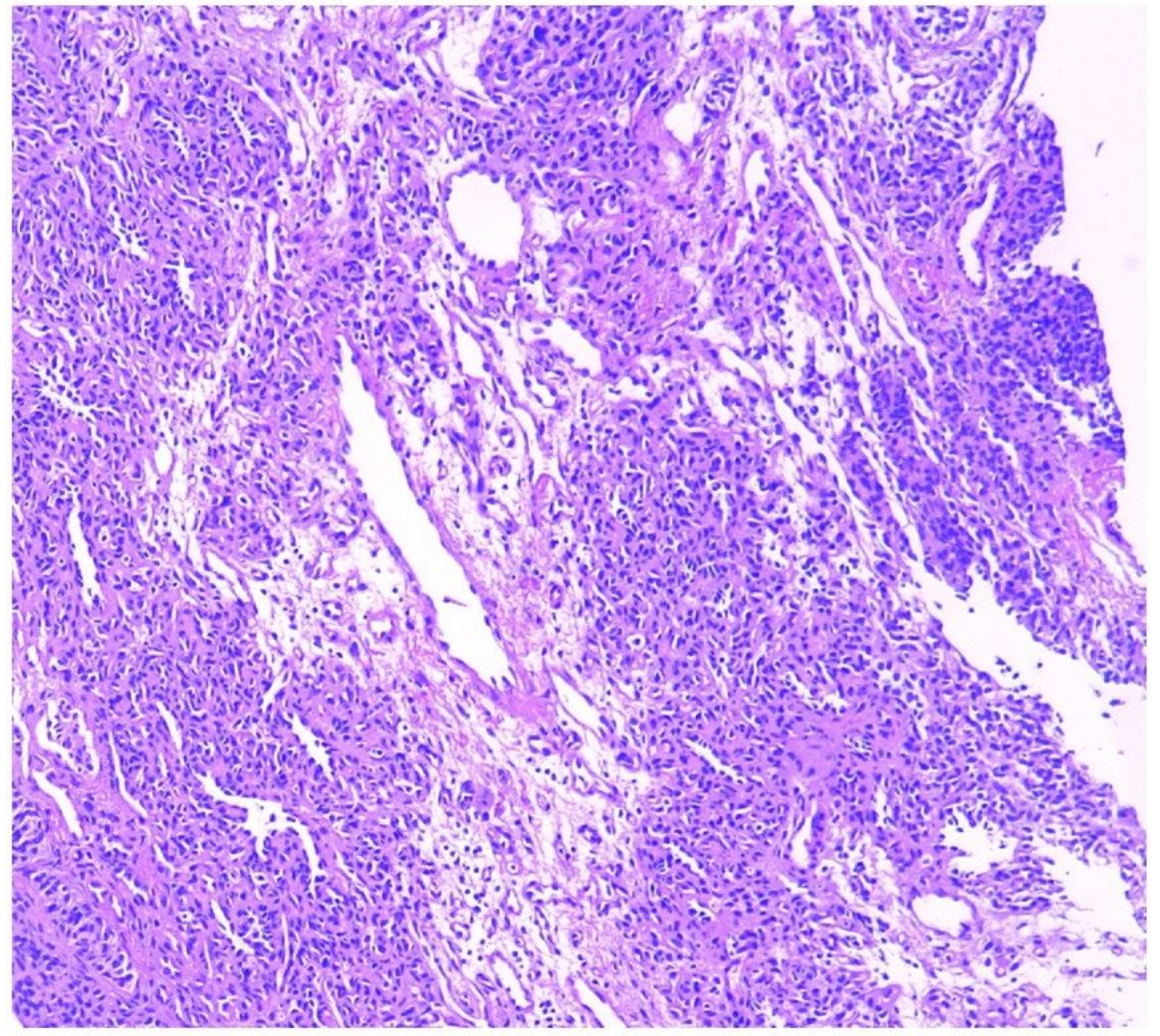

\section{Figure 2}

Hematoxylin and eosin staining revealed a proliferation of capillary-size vessels in the testis parenchyma. 